\title{
Finite Element Method on Contact Problem of Non-newtonian Fluid Material
}

\author{
Lu-Lu Ma, Lei Hou \\ Department of Mathematics, Shanghai University, Shanghai, China \\ E-mail: amandalulu918@163.com, houlei@shu.edu.cn
}

\begin{abstract}
In this paper, we use finite element method to study contact problem of non-Newtonian fluid material. We focus on Cauchy equation which describes the velocity changes in the flow field. The space domain is discrete by Lagrange interpolation function with 16-point bicubic elements. The time domain is discrete by two schemes: two-step Adams explicit scheme and Crank-Nicolson scheme. We study the convergence. In numerical experiments, the error of the equation is shown by comparing the numerical solutions with the exact solutions.
\end{abstract}

Keywords-non-Newtonian fluid; finite element method; contact problem; two-step Adams explicit scheme

\section{INTRODUCTION}

With the continuous development of network and modern communication technology, the concept of the networked intelligent sensor based on the technology of wireless produced. In some special goods transport, environmental parameters have higher requirements for container. Wireless sensor network (WSN) is a good way to monitoring parameters. We apply WSN to warehouse management. Three dimensional space deployment of the wireless sensor network nodes is one of the big problems in the research. One of the effective node deployment plan, is to perform finite element meshes based on cube or sphere on the certain storage space.

Under the condition of high speed collision, the solid material can cause enormous deformation in such a short time. Thus, we can agree that it also has fluid property. Socalled non-Newtonian fluid is a fluid whose shear stress and shear rate cannot always keep a linear relationship, such as blood, toothpaste, oil paint and slurry. Different rheological properties of different type of non-Newtonian fluid will appear under the changes of shear rate. For example, Bingham plastic body exists yield stress. Besides, dilatants fluid has shear thickening properties. With the continuous development of materials science and related research technology, the applications of non-Newtonian fluid material field are deepening.

In the research of non-Newtonian fluid material, we always use coupled PDE equations. The standard P-T/T equation is the best estimates for the stress over-shoot. Cauchy conservation equation may be used to calculate the large deformation resulting from stress (shear thinning). It can be used to describe the velocity and the stress distribution in the contract problem.

In this paper, we focus on the Cauchy equation which describes the velocity changes in the flow field.

$$
\frac{\partial u}{\partial t}=\frac{1}{\rho} \nabla \cdot \tau-(u \cdot \nabla) u .
$$

Here $\rho$ is the density, u is the velocity, and $\tau$ is the stress, and

$$
\begin{aligned}
& u=u_{1}(x, y ; t)+j u_{2}(x, y ; t), \\
& \nabla=i \frac{\partial}{\partial x}+j \frac{\partial}{\partial y}, \tau=\left(\begin{array}{ll}
\tau_{11}(x, y ; t) & \tau_{12}(x, y ; t) \\
\tau_{21}(x, y ; t) & \tau_{22}(x, y ; t)
\end{array}\right), \tau_{12}=\tau_{21} .
\end{aligned}
$$

Then the component equations are as follows:

$$
\begin{aligned}
& \frac{\partial u_{1}}{\partial t}=\frac{1}{\rho}\left(\frac{\partial \tau_{11}}{\partial x}+\frac{\partial \tau_{12}}{\partial y}\right)-\left(u_{1} \frac{\partial u_{1}}{\partial x}+u_{2} \frac{\partial u_{1}}{\partial y}\right), \\
& \frac{\partial u_{2}}{\partial t}=\frac{1}{\rho}\left(\frac{\partial \tau_{12}}{\partial x}+\frac{\partial \tau_{22}}{\partial y}\right)-\left(u_{1} \frac{\partial u_{2}}{\partial x}+u_{2} \frac{\partial u_{2}}{\partial y}\right) .
\end{aligned}
$$

Each equation above includes space variables $(\mathrm{x}, \mathrm{y})$ and time variable t. They would be handled separately. We use the combination method of finite element and difference to solve these equations. Using weighted residuals method, the weak forms of five component equations are obtained. Take the first equation for example.

$$
<\frac{\partial \tilde{u}_{1}}{\partial t}, \psi_{j}>=<\frac{1}{\rho}\left(\frac{\partial \tilde{\tau}_{11}}{\partial x}+\frac{\partial \tilde{\tau}_{12}}{\partial y}\right)-\left(\tilde{u}_{1} \frac{\partial \tilde{u}_{1}}{\partial x}+\tilde{u}_{2} \frac{\partial \tilde{u}_{1}}{\partial y}\right), \psi_{j}>
$$

So the solving problems of the component equations change to find weak solutions: ũ1, ũ2, $\tilde{\tau}_{11}, \tilde{\tau}_{12}$.

\section{THE SEMI-DISCRETIZATION OF THE SPACE}

\section{A. The Choice of Shape Functions}

Rectangular type regions occur in many problems in physics and engineering, ensuring an important role in the finite element method for the rectangular element. We use Lagrange interpolation to construct shape functions. Bicubic shape functions of 16 nodes on the standard cell $e=[-1,1]$ $\times[-1,1]$ are as follows:

$$
\begin{gathered}
\varphi_{i}(\xi, \eta)=\left(1+\xi_{i} \xi\right)\left(1+\eta_{i} \eta\right)\left(1-9 \xi^{2}\right)\left(1-9 \eta^{2}\right) / 256, i=1,2,3,4 \\
\varphi_{i}(\xi, \eta)=-9\left(1+\xi_{i} \xi\right)\left(1+9 \eta_{i} \eta\right)\left(1-9 \xi^{2}\right)\left(1-\eta^{2}\right) / 256, i=7,8,11,12 \\
\varphi_{i}(\xi, \eta)=-9\left(1+\eta_{i} \eta\right)\left(1+9 \xi_{i} \xi\right)\left(1-\xi^{2}\right)\left(1-9 \eta^{2}\right) / 256, i=5,6,9,10 \\
\varphi_{i}(\xi, \eta)=81\left(1+9 \eta_{i} \eta\right)\left(1+9 \xi_{i} \xi\right)\left(1-\xi^{2}\right)\left(1-\eta^{2}\right) / 256, i=13,14,15,16 .
\end{gathered}
$$

The natural coordinates of these 16 nodes are marked as (see Figure. 1) 


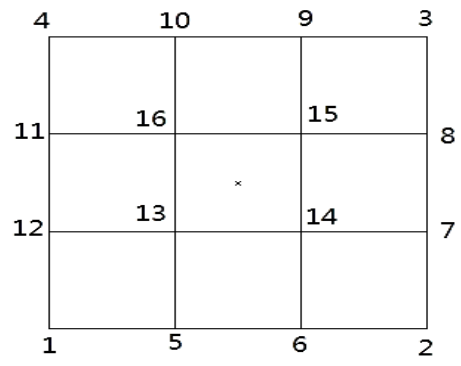

Figure 1. Mesh generation.

$$
\begin{aligned}
\left\{\xi_{i}\right\}^{T}= & \{-1,1,1,-1,-1 / 3,1 / 3,1,1,1 / 3,-1 / 3,-1,-1, \\
& -1 / 3,1 / 3,1 / 3,-1 / 3\} ; \\
\left\{\eta_{i}\right\}^{T}= & \{-1,-1,1,1,-1,-1,-1 / 3,1 / 3,1,1,1 / 3,-1 / 3, \\
& -1 / 3,-1 / 3,1 / 3,1 / 3\},
\end{aligned}
$$

Let $\psi \mathrm{i}=\varphi \mathrm{i}, \mathrm{Vh}=\operatorname{span}\{\varphi 1(\mathrm{x}, \mathrm{y}), \ldots, \varphi 16(\mathrm{x}, \mathrm{y})\}$, where $\varphi \mathrm{i}(\mathrm{x}, \mathrm{y})(\mathrm{i}=1, ., 16)$ is the basis of test function space.

\section{B. The Convergence of Spatial Discretization}

Consider the auxiliary problem (I):

$$
\left\{\begin{array}{l}
\frac{\partial u}{\partial t}=g-(u \cdot \nabla) u, \\
\left.u\right|_{\Gamma}=0,
\end{array}\right.
$$

where

$$
u=\left(\begin{array}{l}
u_{1}(x, y ; t) \\
u_{2}(x, y ; t)
\end{array}\right), g=\left(\begin{array}{l}
g_{1}(x, y ; t) \\
g_{2}(x, y ; t)
\end{array}\right),(x, y) \in \Omega, t \in[0, T] .
$$

For any $t \in[0, T]$, let $u(x, y ; t)$ be the genuine solution of Problem (I), and simply marked as $u(t)$. Let $V_{h}$ be test function space. Consider the variation problem equivalent to Problem (I):

$$
\left(\frac{\partial u(t)}{\partial t}, \nabla v\right)+((u \cdot \nabla) u, \nabla v)=(g, \nabla v), \quad \forall v \in V_{h}
$$

Let $u_{h}(t)$ be the finite element solution of Problem (I). Usually, we use Gauss quadrature to calculate the energy integral. Thus the error e in numerical calculation exists inevitably. Set the actual result to be $\hat{u}_{h}(t)$, then $\hat{u}_{h}(t)=u_{h}(t)+e$.

To sum up, for any $t \in[0, T]$, the error which is brought from finite element spatial discretization is

$$
R_{F E M}(t)=\left\|\hat{u}_{h}(t)-u(t)\right\| \leq\left\|u_{h}(t)-u(t)\right\|+\|e\| .
$$

Let $u_{L}$ be the basic function expression, which take Lagrange bicubic shape functions as basis. We introduce the conclusion of interpolation precision first.

Lemma 1 Suppose $\mathrm{u}$ is sufficiently smooth, then there exists a $\mathrm{C} \geq 0$, such that

$$
\left\|\nabla\left(u-u_{L}\right)\right\| \leq C h^{3} \text {. }
$$

Then we introduce projection operator $\mathrm{P}: H \rightarrow V_{h}$, for $\forall v \in V_{h}$

$$
(\nabla(P u-u), \nabla v)=0
$$

The projection operator satisfies the following condition: Lemma 2 Let $\mathrm{u}$ be the generalized solution of Problem (I), $u_{L}$ be the basic function expression, and $\mathrm{P}$ be the projection operator which is defined as equation (3). Then

$\|\nabla(P u-u)\| \leq\left\|\nabla\left(u-u_{L}\right)\right\|$.

Proof For any $v \in V_{h}, P u-v$ also belongs to $V_{h}$. Then

$\|\nabla(P u-u)\|^{2}=(\nabla(P u-u), \nabla(P u-u))$

$\leq(\nabla(P u-u), \nabla(P u-u))+(\nabla(P u-v), \nabla(P u-v))$

$=(\nabla(P u-u), \nabla(P u-v+v-u))+(\nabla(P u-v), \nabla(P u-u+u-v))$

$=(\nabla(P u-u), \nabla(v-u))+(\nabla(P u-v), \nabla(u-v))$

$=(\nabla(u-v), \nabla(u-v))=\|\nabla(u-v)\|^{2}$.

Set $v=u_{L}$, then $\|\nabla(P u-u)\| \leq\left\|\nabla\left(u-u_{L}\right)\right\|$

From lemma 2 we change the error estimate problem between the projection $\mathrm{Pu}$ of the generalized solution on the test function space and the generalized solution, to the approximation problem of $u_{L}$ to $\mathrm{u}$. Furthermore we can prove that this approximation problem can be estimated by interpolation precision.

Theorem 1 Let $\mathrm{u}$ be the generalized solution of Problem (I), $u_{L} \in V_{h}$ be the basic function expression of finite element solution, and $u_{h}$ be the finite element solution of equation (2), then

$$
\left\|u-u_{h}\right\| \leq C h^{-1}\left\|\nabla\left(u-u_{L}\right)\right\| .
$$

Proof Applying Green formula, we have

$$
\left(\nabla \frac{\partial u(t)}{\partial t}, v\right)+((u \cdot \nabla) u, \nabla v)=-(g, \nabla v), \quad \forall v \in V_{h} .
$$

This equation is workable to $u_{h}$

$$
\left(\nabla \frac{\partial u_{h}(t)}{\partial t}, v\right)+\left(\left(u_{h} \cdot \nabla\right) u_{h}, \nabla v\right)=-(g, \nabla v)
$$

Let $\quad u_{h}(t)-u(t)=\left[u_{h}(t)-P u(t)\right]+[P u(t)-u(t)]=\alpha+\beta$.

From (3), (4), and (5), for any $\chi \in V_{h}$, we have

$$
\begin{aligned}
& \left(\nabla \frac{\partial \alpha}{\partial t}, \chi\right)-\left(\left(u_{h} \cdot \nabla\right) \alpha, \nabla \chi\right) \\
= & \left(\nabla \frac{\partial u_{h}}{\partial t}, \chi\right)-\left(\nabla \frac{\partial P u}{\partial t}, \chi\right)-\left(\left(u_{h} \cdot \nabla\right) u_{h}, \nabla \chi\right)+\left(\left(u_{h} \cdot \nabla\right) P u, \nabla \chi\right) \\
= & \left(\nabla \frac{\partial u}{\partial t}, \chi\right)-((u \cdot \nabla) u, \nabla \chi)-\left(\nabla \frac{\partial P u}{\partial t}, \chi\right)+\left(\left(u_{h} \cdot \nabla\right) P u, \nabla \chi\right) \\
= & \left(-\nabla \frac{\partial \beta}{\partial t}, \chi\right)-((u \cdot \nabla) u, \nabla \chi)+\left(\left(u_{h} \cdot \nabla\right) P u, \nabla \chi\right) \\
= & \left(-\nabla \frac{\partial \beta}{\partial t}, \chi\right)-((u \cdot \nabla) u, \nabla \chi)+\left(\left(u_{h} \cdot \nabla\right) P u, \nabla \chi\right)-((u \cdot \nabla) P u, \nabla \chi) \\
& +((u \cdot \nabla) P u, \nabla \chi) \\
= & \left(-\nabla \frac{\partial \beta}{\partial t}, \chi\right)+\left(\left[\left(u_{h}-u\right) \cdot \nabla\right] P u, \nabla \chi\right) .
\end{aligned}
$$

Thus 
$\left(\nabla \frac{\partial \alpha}{\partial t}, \chi\right)=\left(-\nabla \frac{\partial \beta}{\partial t}, \chi\right)+\left(\left[\left(u_{h}-u\right) \cdot \nabla\right] P u, \nabla \chi\right)+\left(\left(u_{h} \cdot \nabla\right) \alpha, \nabla \chi\right)$.

Then we estimate each part in the above equation. From Poincare inequality and inverse inequality in the finite element space, we know that for any $\chi \in V_{h}$, there exist $C_{1}, C_{2} \geq 0$, such that

$$
C_{1}\|\chi\| \leq\|\nabla \chi\| \leq C_{2} h^{-1}\|\chi\|
$$

Thus $\left(\left(u_{h} \cdot \nabla\right) \alpha(t), \nabla \chi\right) \leq C_{2} \mu h^{-1}\|\nabla \alpha\|\|\chi\|$, where $\mu \geq\left|u_{h}\right|$. From (6) and the boundedness of operator $\nabla P$, we have

$$
\left(\left[\left(u_{h}-u\right) \cdot \nabla\right] P u(t), \nabla \chi\right) \leq M C_{2} h^{-1}(\|\alpha\|+\|\beta\|)\|\chi\|,
$$

Where $\mathrm{M}$ is the boundary of the operator $\mathrm{P}$ which is unrelated to h. Thus

$\left(\nabla \alpha_{t}, \chi\right) \leq\left\|\nabla \beta_{t} \mid\right\| \chi\left\|+C_{2} \mu h^{-1}\right\| \nabla \alpha\|\| \chi\left\|+M C_{2} h^{-1}(\|\alpha\|+\|\beta\|)\right\| \chi \|$.

Because of (6), there exists $C_{3} \geq 0$, such that

$\left(\nabla \alpha_{t}, \chi\right) \leq C_{3}\left(\left\|\nabla \beta_{t}\right\|+h^{-1}\|\nabla \alpha\|+\|\nabla \beta\|\right)\|\chi\|$.

Set $\chi=\nabla \alpha$. Since $\nabla$ and $\frac{\partial}{\partial t}$ are commutative, we have

$\frac{\partial}{\partial t}\left(\|\nabla \alpha\|^{2}\right) / 2 \leq C_{3}\left(\left\|\nabla \beta_{t}\right\|+h^{-1}\|\nabla \alpha\|+\|\nabla \beta\|\right)\|\nabla \alpha\|$

$$
\leq C_{3}\left(\left\|\nabla \beta_{t}\right\|^{2}+h^{-2}\|\nabla \alpha\|^{2}+\|\nabla \alpha\|^{2}+\|\nabla \beta\|^{2}\right) / 2 .
$$

$\operatorname{Suppose}^{h \leq 1}$, then there exists $C_{4} \geq 0$, such that $C_{1}\|\nabla \alpha\|^{2} \leq C_{4} h^{-2}\left(\left\|\nabla \beta_{t}\right\|^{2}+\|\nabla \alpha\|^{2}+\|\nabla \beta\|^{2}\right)$.

Due to Gronwall inequality and (6), there exists $C \geq 0$, such that

$$
\|\alpha(t)\|^{2} \leq\|\nabla \alpha(t)\|^{2} / C_{1} \leq C h^{-2} \int_{0}^{t}\left(\|\nabla \beta\|^{2}+\left\|\nabla \beta_{t}\right\|^{2}\right) d s
$$

Then

$$
\left\|u_{h}(t)-u(t)\right\| \leq\|\alpha(t)\|+\|\beta(t)\| \leq\|\beta(t)\|+\sqrt{C h^{-2} \int_{0}^{t}\left(\|\nabla \beta\|^{2}+\left\|\nabla \beta_{t}\right\|^{2}\right) d s} .
$$

Thanks to Lemma 2, we can obtain the conclusion of Theorem 1.

\section{THE DISCRETIZATION OF TIME}

Now we consider the difference about the time. In the previous papers, we have discussed the convergence of Euler and Crank-Nicolson scheme. In this paper, we focus on the two-step Adams explicit scheme. Accordingly, the scheme of (1) is

$$
u^{n+1}=u^{n}+3 \Delta t\left[g^{n}-u^{n}\left(f\left(u^{n}\right)\right] / 2-\Delta t\left[g^{n-1}-u^{n-1}\left(f\left(u^{n-1}\right)\right] / 2 .\right.\right.
$$

For the convenience of discussion, (1) could be rewritten as:

$$
\frac{\partial u}{\partial t}=g-\phi(u)
$$

Then the scheme becomes

$$
u^{n+1}=u^{n}+3 \Delta t\left[g^{n}-\phi^{n}\right] / 2-\Delta t\left[g^{n-1}-\phi^{n-1}\right] / 2,
$$

where $g^{n}=g\left(t^{n}\right), \phi^{n}=\phi\left(u^{n}\right)$. We have the following conclusion of the scheme.

Theorem 2 Set $u\left(t^{n}\right), g\left(t^{n}\right)$ be the values of $u, g$ separately when $t=t^{n}=t_{0}+n \Delta t$. Set $u^{n}$ be the approximate solution of (7), and $\tilde{\phi}^{n}=\phi\left(u\left(t^{n}\right)\right)$. Let $\varepsilon^{n}=u\left(t^{n}\right)-u^{n}$

$$
\begin{aligned}
R^{n+1}= & u\left(t^{n+1}\right)-\left\{\left[u\left(t^{n}\right)+3 \Delta t\left[g\left(t^{n}\right)-\widetilde{\phi}^{n}\right] / 2\right.\right. \\
& \left.-\Delta t\left[g\left(t^{n-1}\right)-\widetilde{\phi}^{n-1}\right] / 2\right\},
\end{aligned}
$$

then

$$
|R|=\max _{n}\left\{\left|R^{n}\right|\right\} \leq C \Delta t^{3},|\varepsilon|=\max _{n}\left\{\left|\varepsilon^{n}\right|\right\} \leq C \Delta t^{2} \text {. }
$$

Proof Taking the Taylor expansion, we have

$$
\begin{aligned}
R^{n+1}= & u\left(t^{n+1}\right)-\left[u\left(t^{n}\right)+3 \Delta t u^{\prime}\left(t^{n}\right) / 2-\Delta t u^{\prime}\left(t^{n-1}\right) / 2\right] \\
= & u\left(t^{n}\right)+\Delta t u^{\prime}\left(t^{n}\right)+\Delta t^{2} u^{\prime \prime}\left(t^{n}\right) / 2+\Delta t^{3} u^{\prime \prime \prime}\left(t^{n}\right) / 6 \\
& -\left[u\left(t^{n}\right)+3 \Delta t u^{\prime}\left(t^{n}\right) / 2-\Delta t u^{\prime}\left(t^{n-1}\right) / 2\right] \\
= & -\Delta t u^{\prime}\left(t^{n}\right) / 2+\Delta t^{2} u^{\prime \prime}\left(t^{n}\right) / 2+\Delta t^{3} u^{\prime \prime \prime}\left(t^{n}\right) / 6+\Delta t u^{\prime}\left(t^{n-1}\right) / 2 \\
= & {\left[-\Delta t u^{\prime}\left(t^{n-1}\right) / 2-\Delta t^{2} u^{\prime \prime}\left(t^{n-1}\right) / 2-\Delta t^{3} u^{\prime \prime \prime}\left(t^{n-1}\right) / 4\right] } \\
& +\left[\Delta t^{2} u^{\prime \prime}\left(t^{n-1}\right) / 2+\Delta t^{3} u^{\prime \prime \prime}\left(t^{n-1}\right) / 2\right]+\Delta t^{3} u^{\prime \prime \prime}\left(t^{n}\right) / 6 \\
& +\Delta t u^{\prime}\left(t^{n-1}\right) / 2 \\
= & \Delta t^{3}\left[u^{\prime \prime \prime}\left(t^{n-1}\right) / 4+u^{\prime \prime \prime}\left(t^{n}\right) / 6\right] \\
C= & \max \left\{\left|5 u^{\prime \prime \prime}(t) / 12\right|\right\}, \text { then } \\
|R|= & \max _{n}\left\{\left|R^{n}\right|\right\} \leq C \Delta t^{3}
\end{aligned}
$$

Next we consider $|\varepsilon|$.

$$
\begin{aligned}
\varepsilon^{n+1}-\varepsilon^{n} & =\left[u\left(t^{n+1}\right)-u^{n+1}\right]-\left[u\left(t^{n}\right)-u^{n}\right] \\
& =\left[u\left(t^{n+1}\right)-u\left(t^{n}\right)\right]-\left[u^{n+1}-u^{n}\right] \\
& =\frac{3 \Delta t}{2}\left(\phi^{n}-\widetilde{\phi}^{n}\right)-\frac{\Delta t}{2}\left(\phi^{n-1}-\tilde{\phi}^{n-1}\right)+R^{n+1} .
\end{aligned}
$$

Thus

$$
\left|\varepsilon^{n+1}\right| \leq(1+3 \Delta t L / 2)\left|\varepsilon^{n}\right|-\Delta t L\left|\varepsilon^{n-1}\right| / 2+R .
$$

When we know $u_{0}$, we use Euler scheme to calculate $u^{1}$. Due to $\varepsilon^{1}=u\left(t^{1}\right)-u^{1}$, we have $\left|\varepsilon^{1}\right| \leq C \Delta t^{2}$. And $\varepsilon^{0}=u\left(t_{0}\right)-u_{0}=0$. We can obtain the conclusion of Theorem 2 by recurrence relations (9).

In fact, $R^{n}$ is commonly known as local truncation error, and $\varepsilon^{n}$ is called global truncation error.

\section{THE NUMERICAL ANALYSIS}

Let the type of the solving area and boundary condition be consistent with the original coupling problem. Suppose there exist genuine solution:

$$
u=\left(\begin{array}{l}
u_{1}(x, y ; t) \\
u_{2}(x, y ; t)
\end{array}\right)=\left[\begin{array}{c}
x y t(H-y) / 30+u_{00} y / H \\
x y t(H-y) / 30
\end{array}\right]
$$

Here the stress $\tau$ is regarded as a given value which is a 
random variable and the initial boundary value conditions is $u_{00} y / H$. $\mathrm{H}$ is the height of the solution area of the quadrilateral, ${ }^{u_{00}}$ is the forced boundary conditions of the upper boundary.

TABLE I $\left|u^{n}-u\left(t^{n}\right)\right|$ IN DIFFERENT CONDITION

\begin{tabular}{|c|c|c|c|c|}
\hline \multirow{2}{*}{ Nodes number } & \multicolumn{2}{|c|}{$\Delta t=0.5, h=0.5$} & \multicolumn{2}{|c|}{$\Delta t=0.2, h=0.5$} \\
\hline & Adams & C-N & Adams & C-N \\
\hline 1 & 0.011713 & 0.071331 & 0.007613 & 0.008409 \\
\hline 2 & 0.039092 & 0.023623 & 0.002554 & 0.001842 \\
\hline 3 & 0.03196 & 0.195855 & 0.002346 & 0.002682 \\
\hline 4 & 0.022054 & 0.015055 & 0.003648 & 0.00494 \\
\hline 5 & 0.023163 & 0.029418 & 0.000628 & 0.009134 \\
\hline 6 & 0.00492 & 0.015993 & 0.00085 & 0.003116 \\
\hline 7 & 0.0136 & 0.035904 & 0.004264 & 0.003816 \\
\hline 8 & 0.006602 & 0.03141 & 0.000232 & 0.012383 \\
\hline 9 & 0.015131 & 0.002112 & 0.007195 & 0.004864 \\
\hline 10 & 0.009176 & 0.008373 & 0.002165 & 0.001119 \\
\hline 11 & 0.000137 & 0.02788 & $2.97 \mathrm{E}-05$ & 0.000623 \\
\hline 12 & 0.005763 & 0.018394 & 0.004148 & 0.006556 \\
\hline 13 & 0.00511 & 0.043238 & 0.002888 & 0.004604 \\
\hline 14 & 0.007635 & 0.019367 & 0.0008 & 0.007238 \\
\hline 15 & 0.005126 & 0.008609 & 0.003849 & 0.003999 \\
\hline 16 & 0.000147 & 0.035145 & 0.000836 & 0.011232 \\
\hline 17 & 0.022375 & 0.002983 & 0.000542 & 0.001747 \\
\hline 18 & 0.010451 & 0.007661 & 0.009267 & 0.001303 \\
\hline 19 & 0.004088 & 0.041877 & 0.006715 & 0.004296 \\
\hline 20 & 0.010862 & 0.020659 & 0.001821 & 0.003138 \\
\hline 21 & 0.001644 & 0.018997 & 0.000779 & 0.010849 \\
\hline 22 & 0.014215 & 0.005447 & 0.002324 & 0.000975 \\
\hline 23 & 0.012014 & 0.014122 & 0.000786 & $8.99 \mathrm{E}-05$ \\
\hline 24 & 0.009609 & 0.019329 & 0.000338 & 0.001486 \\
\hline 25 & 0.006997 & 0.017502 & 0.001113 & 0.006532 \\
\hline 26 & 0.000868 & 0.022252 & 0.004088 & 0.000228 \\
\hline 27 & 0.005638 & 0.016075 & 0.001809 & 0.00327 \\
\hline 28 & 0.012787 & 0.061306 & 0.006044 & 0.006841 \\
\hline 29 & 0.011246 & 0.007276 & 0.005676 & 0.007514 \\
\hline 30 & 0.00586 & 0.021493 & $3.5 \mathrm{E}-05$ & 0.005596 \\
\hline 31 & 0.005989 & 0.008605 & 0.008087 & 0.002162 \\
\hline 32 & 0.012357 & $2.6 \mathrm{E}-05$ & 0.002113 & 0.000516 \\
\hline 33 & 0.00787 & 0.052767 & 0.006389 & 0.027963 \\
\hline 34 & 0.008911 & 0.017883 & 0.00238 & 0.007039 \\
\hline 35 & 0.013104 & 0.00132 & 0.002964 & 0.00096 \\
\hline
\end{tabular}

In the numerical experiments, we adopt Lagrange interpolation function with 16-point bicubic elements on the space; two-step Adams explicit and Crank-Nicolson scheme on the time. Consider the $2 \times 2$ grid. Let the lower boundary of the grid be fixed on the $x$-axis. There is a velocity along the positive direction of $x$-axis at the upper boundary. Set $u_{00}=0.01$. The numerical results of $\left|u^{n}-u\left(t^{n}\right)\right|$ are listed as follows.

Under the different schemes, Table I shows the errors of the numerical and exact aid-solution with $\Delta t=0.5$ and $\Delta t=0.2$ at $h=0.5$ respectively. It can be seen that error norm calculated by two-step Adams explicit scheme is smaller than that by Crank-Nicolson scheme. Moreover, when $\Delta t$ is smaller, the error norm is smaller.

\section{ACKNOWLEDGMENT}

The authors would like to thank the editor, reviewers, and persons who improve the results of this paper. The paper is supported by NSFC 11271247.

\section{REFERENCES}

[1] Y. Peng, B.-H. Li, H.-B. Ji, L. Sun, C.-C. Dong, and J.-L. Yuan, "Application and Prospect of non-Newtonian Fluid in the Industrial Field," Light Industry Machinery, vol. 32, no. 1, 2014.

[2] K.-T. Li and A.-X. Huang, "The finite element method and its application," Beijing: Science Press, 2006.

[3] F. Brezzi, "On the existence, uniqueness and approximation of saddlepoint problems arising from Lagrangian multipliers," RAIRO Annl, vol. 8, no. R-2, pp. 129-151, 1974.

[4] F. Brezzi and M. Fortin, "Mixed and hybrid finite element methods," Numerical Methods for Partial Differential Equations, vol. 23, no. 4, pp.785-797, 1991.

[5] L.-H. Wang and X.-J. Xu, "The Mathematical Bases of Finite Element Methods," Beijing: Science Press, 2004.

[6] Q.-D. Zhu, "High precision post-processing theory of finite element", Beijing: Science Press, 2008

[7] L. Hou and R. Harwood, "Non-linear properties in Newtonian and non-Newtonian equations," Nonlinear Analysis: Theory, Method \& Applications, vol. 30, no. 4, pp. 2497-2505, 1997.

[8] L. Hou and L. Cai, "Nonlinear Property of the visco-elastic-plastic material in the impact problem," Journal of Shanghai University (English Edition), vol.13, no. 1, pp. 23-28, 2009.

[9] Q. Lin and N. Yan, "Global super-convergence for Maxwell's equation," Mathematics of Computation, vol. 69, no. 229, pp. 159-176, 2000.

[10] L. Hou, J.-J. Zhao and H.-L. Li, "Finite element convergence analysis of two-scale non-Newtonian flow problems", Advances Materials Research, vol. 718-720, pp. 1723-1728, 2013.

[11] D. Bai, L. Zhang, "The finite element method for the coupled Schrodinger-KdV equations", Physics Letters A, pp. 2237-2244, 2009

[12] A.-R. Mitchell and R. Wait, "The finite element method in partial differential equations", Wiley, New York, 1977

[13] Q.-Y. Li, N.-C. Wang and D.-Y. Yi, "Numerical Analysis", Beijing: Tsinghua University Press, 2008.

[14] J.-Z. Zhu, R.-L. Taylor and O.-C. Zienkiewicz, "The finite element method: its basis and fundamentals", Butterworth-Heinemann, 2005. 